\title{
A Novel Measurement System for Cardiac Flow Analysis Applied to Phase Contrast Magnetic Resonance Imaging of the Heart
}

\author{
K.K.L. Wong ${ }^{1}$, R.M. Kelso ${ }^{2}$, S.G. Worthley ${ }^{3}$, P. Sanders ${ }^{3}$, J. Mazumdar ${ }^{1}$, and D. Abbott ${ }^{1}$ \\ ${ }^{1}$ Centre for Biomedical Engineering/School of Electrical \& Electronic Engineering, University of Adelaide, Adelaide, Australia \\ ${ }^{2}$ School of Mechanical Engineering, University of Adelaide, Adelaide, Australia \\ ${ }^{3}$ Cardiovascular Research Centre/School of Medicine, University of Adelaide, Adelaide, Australia
}

\begin{abstract}
Velocity-encoded magnetic resonance imaging of normal subjects is performed to produce flow fields of blood in right atrium. The aim of this experiment is to demonstrate the state of change in swirling of blood within a cardiac chamber using a flow imaging modality, and to quantify it for clinical applications. Velocity maps that are displayed using streamline tracings illustrate the flow behavior at various cardiac phases. Rotation of blood can be observed in the atrium, and we have developed a statistical based system for characterizing the strength of the vortices. From our study, we are able to acquire an indication of the changes in vorticity magnitude during one cardiac cycle with the support of vorticity fields based on the cardiac flow. This has improved our understanding of blood motion within the heart chamber which may have implications in blood circulation efficiency.
\end{abstract}

Keywords-Phase contrast magnetic resonance imaging, Velocity-encoding, Intra-cardiac flow, Vorticity.

\section{INTRODUCTION}

Phase contrast magnetic resonance imaging (MRI) allows velocity mapping based on the intrinsic sensitivity of MRI to flow, and enables the acquisition of spatial registered functional information simultaneously with morphological information [1]. Three-dimensional MRI-based velocity mapping operates by registering three separate flowsensitive volumes in the $x, y$ and $z$ orientations of the scan. The flow velocities may be computed by determining the shift of phase pertaining to the collection of imaged blood proton spins and reconstructing the flow vectors in advanced visualization packages. This concept has varying terminologies in literature, the most common being phase contrast MRI, while some studies labeled it as phase velocity MRI [2]. In general, such MRI based techniques form a class of approach known as magnetic resonance velocimetry (MRV) or sometimes also called magnetic resonance image velocimetry (MRIV), and has been very commonly used for producing visualization and investigation of flows even within non-organic structures [3].

Further development of phase contrast MRI to produce multi-slice cine images involves ECG synchronized timeresolved framework to allow assessment of blood-flow characteristics with high spatial and temporal resolution of a cardiovascular region of interest (ROI) [4,5]. Recent development on navigator-gated time-resolved cine phase contrast MRI can control image alterations due to respiration of patient during scans and the resulting ghosting effects.

The use of phase contrast MRI applied onto cardiac imaging of right atria enables a good assessment of vortices that exist in the cardiac chamber. Vortical flow behavior is essential in efficient heart operation and blood circulation $[6,7]$. Previously, the study of vortices in the human heart has also been performed using phase contrast data [8]. In particular, study of vortices in the left atrium has been performed using three-dimensional phase contrast MRI $[6,9,10,11]$.

The superiority of MRI over other imaging modalities is the capability of generating up to three-dimensional velocity profiles that can reflect the dynamics of blood flow more accurately and with quantifiable details. Apart from such localised quantification that can provide interactive visualization in cardiovascular flow [5], phase contrast MRI has also been utilised in global flow quantification such as determination of flow volumes in arterial structures, and in particular, blood ejection volumes in the ascending aorta $[2,12]$ as well as arterial wall shear stress [13]. It has been widely documented that phase contrast MRI is an established flow-imaging scheme for cardiovascular examination of human subjects and compares well with ultrasound technology [12].

In this paper, we examine the implementation of phase contrast MRI on a case study subject. In our literature review, we examine the current technologies that perform medical imaging of the heart, particularly flow visualization. The methodology section introduces the building blocks and mathematics that enable the performance of the cardiac flow visualization. Experimental procedures are then stated, and our results present the velocity and vorticity flow maps of the right atrium of the subject. Next, we present and discuss some results of the flow imaging from this study. Finally a conclusion is made based on the implications of the flow imaging framework. 


\section{ReVIEW OF CURRENT TECHNOLOGIES}

\section{A. Color Doppler Sonography}

Doppler ultrasound is based on Doppler shift caused by blood scatter movement [14], and is a widely accepted technique to visualize blood flow patterns. Analysis of the flow field obtained by ultrasound is useful in cardiac diagnosis. However, the output from the Doppler ultrasound method is usually represented as a two-dimensional image.

Real-time blood motion imaging using color sonograms can be utilized. For this medical imaging modality, the speckle pattern from the blood flow signal is preserved, enhanced, and visualized.

\section{B. Phase Contrast Magnetic Resonance Imaging}

Phase contrast signals can be represented using images. The intensity of each pixel corresponds to the blood velocity at the measured location. To quantify a velocity in one spatial dimension, at least two phase images have to be taken for subtraction of flow-induced phase shift from background phases caused by susceptibility-induced nonhomogeneities and coil sensitivity changes [15]. The construction of a three-dimensional flow vector can be carried out using such a technique as shown in Fig. 1.

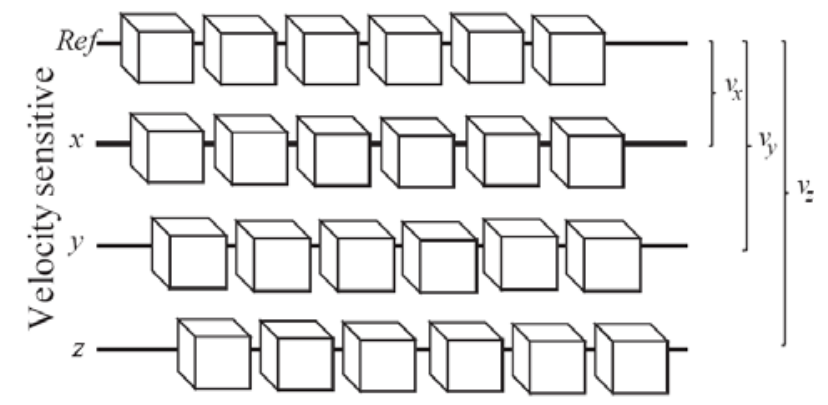

Fig. 1 Phase contrast MRI velocimetry. Velocities $v_{x}, v_{y}$ and $v_{z}$ can be calculated by subtraction of spin phase of measured volumes with that of the reference spaces $R e f$

\section{Methodology}

\section{A. Flow Grid Representation}

A dense velocity field (one velocity vector per pixel) is generated using phase contrast MRI, and a vector averaging for sampling window resolution of 3 by 3 pixels is carried out. Calibration is carried out with respect to all the values present in the displayed phases of the cardiac cycle to improve visualization and referencing.

\section{B. Vorticity Field Representation}

Vorticity map is generated from the flow field [16]. The vorticity flow values which appeared per slice have their magnitudes scaled. Positive values signify counterclockwise $(\mathrm{CCW})$ rotation, whereas negative values represent clockwise $(\mathrm{CW})$ motion of the blood. Therefore, the magnitudes of these values give an indication of the angular velocity and their polarity signifies the direction of the rotation. These may be represented by a color scale with maximum $\mathrm{CCW}$ and $\mathrm{CW}$ vorticity magnitudes, which correspond to red and blue respectively.

\section{EXPERIMENTS}

\section{A. Case Study and Investigation Procedure}

In this study, we imaged the right atrium of a 22 years old male subject using velocity-encoded (VENC) MRI.

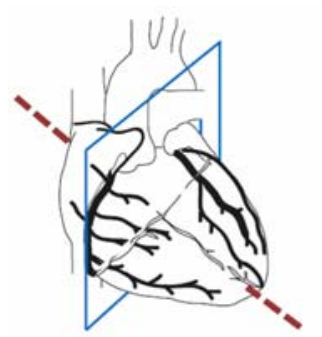

Fig. 2 MRI scan through heart of normal subject. The scanning of the heart is based on the two-chamber short axis orientation

A scan is performed at the section of the heart where the atria are positioned. The scan section is taken at a location shown in Fig. 2 whereby the scan is perpendicular to an axis joining the top of the heart to the apex through the septum. Table I presents the configuration of the MRI scans.

\section{B. Phase Contrast MRI Scan Procedure}

For this purpose, velocity-encloded MRI was performed using a Siemens Sonata, 1.5 Tesla, model-syngo MR 2004A scanner with Numaris - 4, Series No: 21609 software. Cine-MRI was performed using one slice in short axis views through the atria. All images were acquired with retrospective gating and 25 phases.

\section{Parameters for Data Analysis}

The histogram of a vorticity map with vorticity values in the range $[0, L-1]$ is a discrete function $h\left(r_{k}\right)=n_{k}$, where $r_{k}$ is the $k$ th vorticity value and $n_{k}$ is the number of pixels in the flow map having vorticity value $r_{k}$ [17].Statistical 
Table 1 MRI and vorticity measurement properties. The scan properties of phase contrast MRI are presented here. The vorticity flow maps can be determined from this information. These parameter values are used to calibrate these flow maps, and indicating the slice resolutions

\begin{tabular}{clcc} 
PHASE CONTRAST MRI SCAN & & \\
\hline Symbol & Quantity & 1 & Units \\
\hline$p$ & Pixel spacing & 1.67 & $\mathrm{~mm} /$ pixel \\
$t_{s}$ & Trigger time interval & 35.72 & $\mathrm{~ms}$ \\
$s$ & Slice thickness & 6 & $\mathrm{~mm}$ \\
\hline
\end{tabular}

\begin{tabular}{cccc}
\hline \multicolumn{2}{l}{ VORTICITY MEASUREMENT PARAMETERS } \\
\hline Symbol & Quantity & Value & Units \\
\hline$X$ & Image width & 120 & pixel \\
$Y$ & Image height & 150 & pixel \\
$W_{v}$ & Velocity interrogation window & $3 \times 3$ & pixel \\
& &
\end{tabular}

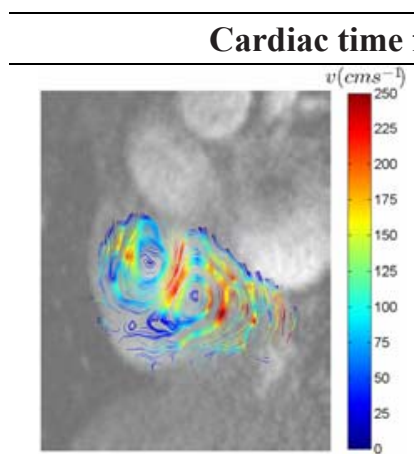

(i) $t=8,\left[\begin{array}{l}\bar{\omega} \\ \sigma\end{array}\right]_{\mu}=\left[\begin{array}{c}3.33 \\ 12.09\end{array}\right] \mathrm{s}^{-1},\left[\begin{array}{c}\bar{\omega} \\ \sigma\end{array}\right]_{m}=\left[\begin{array}{c}0.00 \\ 12.54\end{array}\right] \mathrm{s}^{-1}$

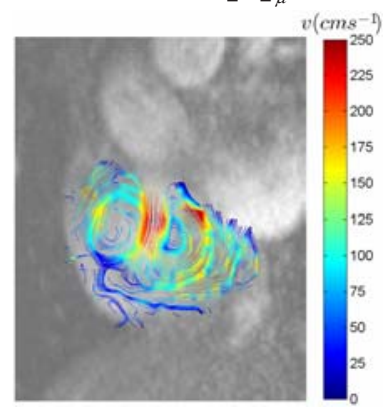

(ii) $t=9,\left[\begin{array}{c}\bar{\omega} \\ \sigma\end{array}\right]_{\mu}=\left[\begin{array}{c}2.59 \\ 11.13\end{array}\right]^{-1},\left[\begin{array}{c}\bar{\omega} \\ \sigma\end{array}\right]_{m}=\left[\begin{array}{c}0.00 \\ 11.43\end{array}\right] \mathrm{s}^{-1}$

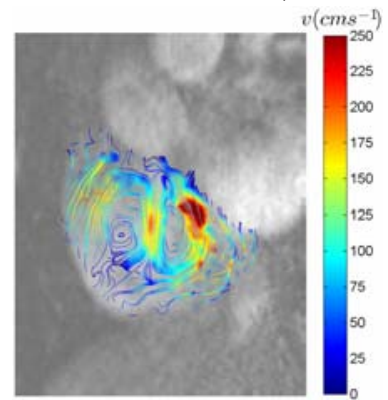

(iii) $t=10,\left[\begin{array}{l}\bar{\omega} \\ \sigma\end{array}\right]_{\mu}=\left[\begin{array}{l}0.23 \\ 9.59\end{array}\right] \mathrm{s}^{-1},\left[\begin{array}{c}\bar{\omega} \\ \sigma\end{array}\right]_{m}=\left[\begin{array}{c}-1.75 \\ 9.79\end{array}\right] \mathrm{s}^{-1}$
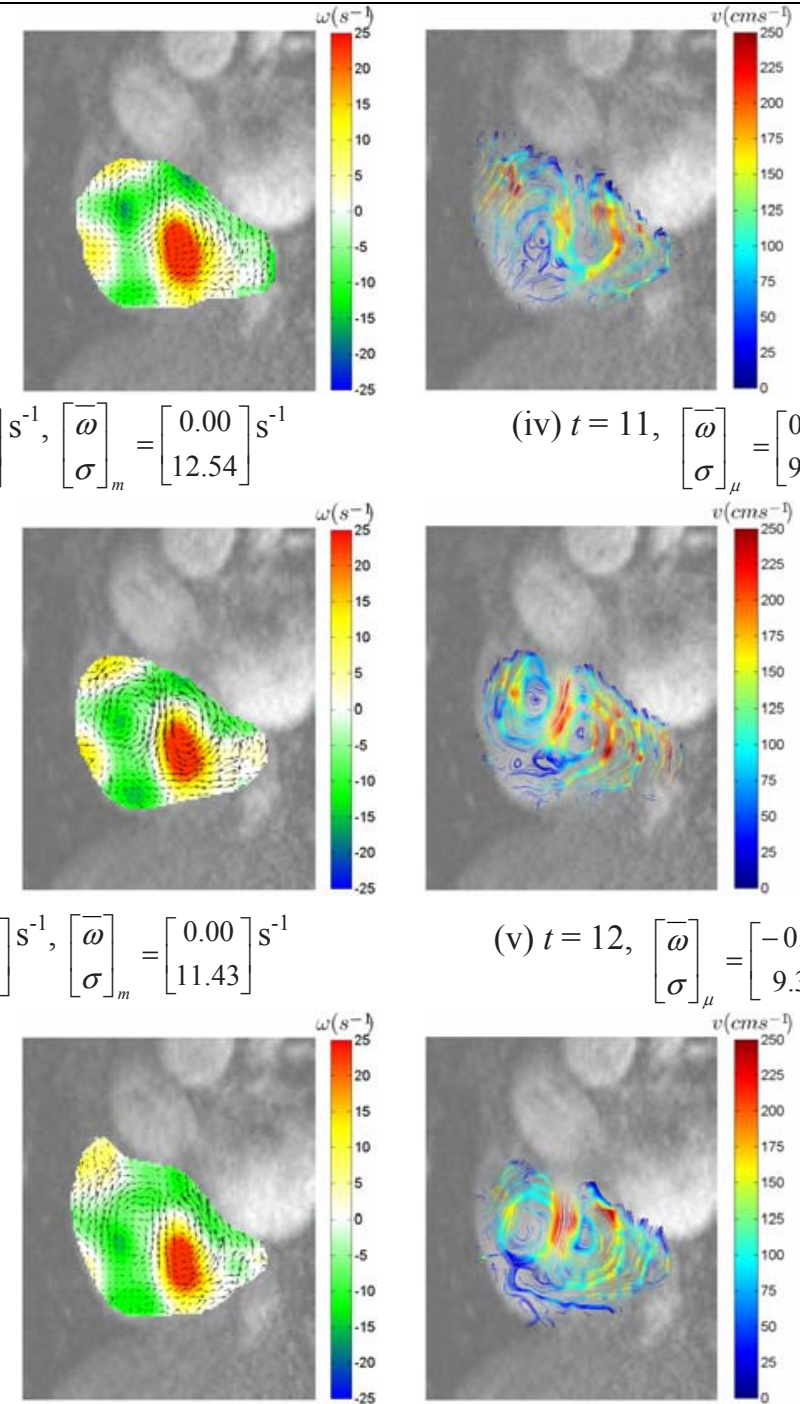

(vi) $t=13,\left[\begin{array}{l}\bar{\omega} \\ \sigma\end{array}\right]_{\mu}=\left[\begin{array}{c}-0.6 \\ 7.13\end{array}\right]^{-1},\left[\begin{array}{l}\bar{\omega} \\ \sigma\end{array}\right]_{m}=\left[\begin{array}{c}-3.05 \\ 7.53\end{array}\right] \mathrm{s}^{-1}$

(iv) $t=11,\left[\begin{array}{l}\bar{\omega} \\ \sigma\end{array}\right]_{\mu}=\left[\begin{array}{l}0.02 \\ 9.10\end{array}\right],\left[\begin{array}{c}\bar{\omega} \\ \sigma\end{array}\right]_{m}=\left[\begin{array}{c}-1.87 \\ 9.30\end{array}\right] \mathrm{s}^{-1}$

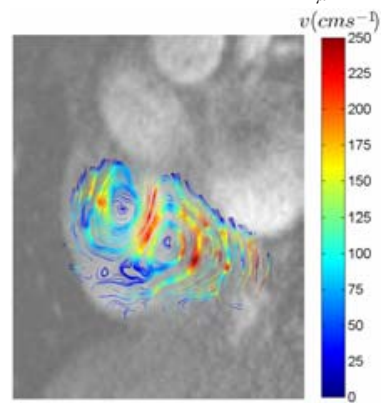

(v) $t=12,\left[\begin{array}{l}\bar{\omega} \\ \sigma\end{array}\right]_{\mu}=\left[\begin{array}{c}-0.66 \\ 9.35\end{array}\right] \mathrm{s}^{-1},\left[\begin{array}{c}\bar{\omega} \\ \sigma\end{array}\right]_{m}=\left[\begin{array}{c}-3.4 \\ 9.74\end{array}\right] \mathrm{s}^{-1}$

$$
\left[\begin{array}{l}
\omega \\
\sigma
\end{array}\right]_{\mu}=\left[\begin{array}{c}
-0.6 \\
7.13
\end{array}\right] \mathrm{S}^{-1},\left[\begin{array}{l}
\bar{\omega} \\
\sigma
\end{array}\right]_{m}=\left[\begin{array}{c}
-3.05 \\
7.53
\end{array}\right] \mathrm{S}
$$
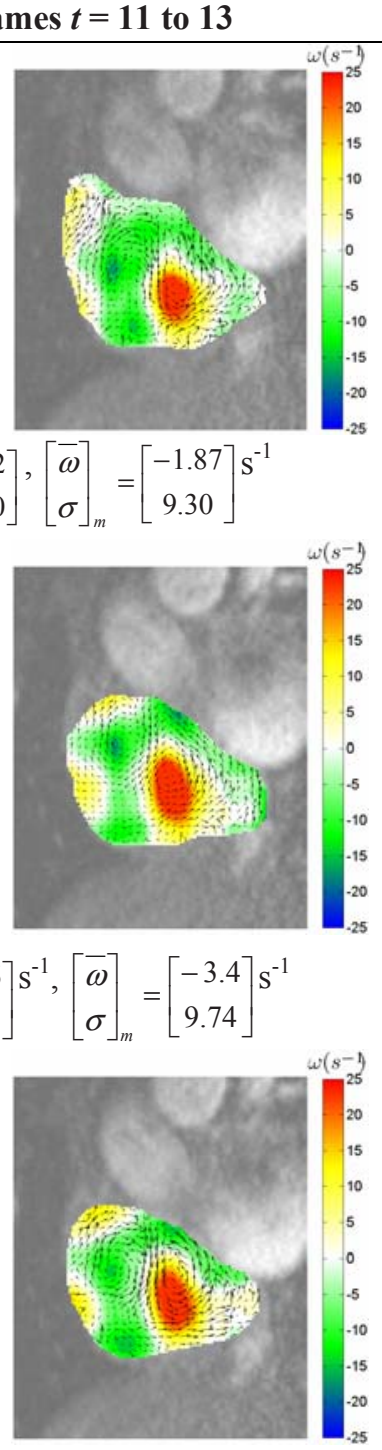

Fig. 3 Flow field visualization of normal right atrium. The visualization of flow in the right atrium of a normal subject is presented for investigation of the vortex behaviour from phase 8 to 13 of one cardiac cycle with 25 phases. Color streamlines, with the color at every patch of the line indicates the magnitude of the velocity (in cms-1) and presents both the direction and speed of flow. Vorticity contour map is superimposed onto the vector plot of each phase to indicate the location and strength of vortices 
quantification of the blood vorticity map in the right atrium is performed by translating all the scalar values.

\section{Results AND DisCUSSION}

We present the flow results and analysis of the right atrium for the selected slice of the heart along the short axis orientation. Histograms are computed for each of these vorticity maps. Note that the mean and median of the histograms are denoted by $\bar{\omega}_{\mu}$ and $\bar{\omega}_{m}$ respectively. Standard deviations with respect to the mean and median are denoted as $\sigma_{\mu}$ and $\sigma_{m}$ respectively. From Fig. 3, based on the streamline plots and vorticity contour maps, we are able to observe a counter-clockwise (CCW) vortex in the atrium along with a clockwise (CW) vortex approximately northwest of it. Vorticity means based on the contour flow maps for six selected phases are characterized by magnitudes of $\bar{\omega}_{\mu}$ from -0.66 to $3.33 \mathrm{~s}^{-1}$. If two vortices of equal strength exist in the chamber simultaneously, $\bar{\omega}_{\mu}$ becomes zero.

The magnitudes of $\sigma$ based on the mean ranges from 7.13 to $12.09 \mathrm{~s}^{-1}$ and are dependent on the characteristic of the flow map. High contrast in vorticity values from the image results in a large standard deviation as compared to images which have vorticity values that do not have a large variance. When there are even number of vortices in the cardiac chamber of analysis, magnitudes of $\bar{\omega}$ cannot be used with confidence as a mode of comparison as the sum of vorticity values for flow in clockwise directions will cancel those in counter-clockwise directions. Using magnitudes of $\sigma$ is a more accurate comparison for the strengths of the vortices.

\section{Conclusions}

We have applied phase contrast MRI on the right atrium of a healthy subject to provide an overview of the swirl patterns present within the blood flow. We have also demonstrated that swirling of blood within the atrium of this subject is overall counter-clockwise.

The description of vortical flow in the right atrium can be concisely presented using velocity and vorticity flow maps. It is important that the flow is correctly calibrated. Useful visualization tools such as contour and streamline plots are utilized in our paper. More importantly, we are also able to characterize the strength of the vortices by compiling histograms of the flow maps and extracting useful statistical properties from them to describe the rotation.

The study has shown that phase contrast MRI is a useful and effective way of visualizing intra-cardiac flow. To the best of our knowledge, this is the first paper that uniquely analyse vorticity within the right atrium. Although we have studied one chamber at this stage, the framework developed in this research can be extended to assess flow in the other heart chambers or cardiovascular structures.

\section{Notes}

Medical image processing software named Medflovan, which is developed by Kelvin KL Wong, is utilized for cardiac flow visualization and analysis in this paper.

\section{REFERENCES}

1. Mark M, Harloff A, Bley TA et al. (2007) Time-resolved 3D MR velocity mapping at 3T: Improved navigator-gated assessment of vascular anatomy and blood flow. J. Magn. Reson. Imaging 25:824-831

2. Powell AJ, Maier SE, Chung T, and Geva T (2000) Phase-velocity cine magnetic resonance imaging measurement of pulsatile blood flow in children and young adults: In vitro and in vivo validation. Pediatr. Cardiol. 21:104-110

3. Elkins CJ, Markl M, Iyengar A, Wicker R, and Eaton J (2004) Full field velocity and temperature measurements using magnetic resonance imaging in turbulent complex internal flows. Int J Heat Fluid Flow 25:702-710

4. Herold V, Morchel P, Faber C, Rommel E, Haase A, and Jakob PM (2006) In vivo quantitative three-dimensional motion mapping of the murine myocardium with PC-MRI at 17.6 T. Magnetic Resonance in Medicine 55:1058-1064

5. Yamashita S, Isoda H, Hirano M et al. (2007) Visualization of hemodynamics in intracranial arteries using time-resolved threedimensional phase-contrast MRI. J. Magn. Reson. Imaging 25:473-478

6. Fyrenius A, Wigström L, Ebbers T et al. (2001) Three dimensional flow in the human left atrium. Heart 86:448-455

7. Pierrakos O, and Vlachos PP (2006) The effect of vortex formation on left ventricular filling and mitral valve efficiency. Journal of Biomechanical Engineering-Transactions of the ASME 128 (4):527-539

8. Yang GZ, Mohiaddin RH, Kilner PJ, and Firmin DN (1998) Vortical flow feature recognition: A topological study of in vivo flow patterns using MR velocity mapping. Journal of Computer Assisted Tomography 22:577-586

9. Ebbers T, Wigström L, Bolger AF et al. (2002) Noninvasive measurement of time-varying three-dimensional relative pressure fields within the human heart. Journal of Biomechanical Engineering 124(3):288-293

10. Fyrenius A, Ebbers T, Wigström L et al. (1999) Left atrial vortices studied with 3D phase contrast MRI. Clinical Physiology and Functional Imaging 19(3), 195

11. Brandt E, Ebbers T, Wigström L et al. (2001) Automatic detection of vortical flow patterns from three-dimensional phase contrast MRI. Proc. Intl. Soc. Mag. Reson. Med 9, 1838

12. Maier SE, Meier D, Boesiger P et al. (1989) Human abdominal aorta: comparative measurements of blood flow with MR imaging and multigated Doppler US. Radiology 171:487-92

13. Oyre S, Pedersen EM, Ringgaard S et al. (1997) In vivo wall shear stress measured by magnetic resonance velocity mapping in the normal human abdominal aorta. Eur. J. Vasc. Endovasc. Surg. 13:263-71

14. Wolbarst AB (1999) Looking Within How X-ray, CT, MRI, Ultrasound, and Other Medical Images are Created, and How They Help Physicians Save Lives University of California Press, Ltd, USA

15. Baltes C, Kozerke S, Hansen MS et al. (2005) Accelerating cine phase-contrast flow measurements using k-t BLAST and k-t SENSE. Magn Reson Med. 54(6):1430-1438

16. Raffel M, Willert C, and Kompenhans J (1998) Particle Image Velocimetry Springer, Germany

17. Gonzalez RC, Woods RE (2002) Digital Image Processing, 2nd edition, Prentice-Hall, Inc., New Jersey, USA 